\title{
Few genes are associated with the capability of pancreatic ductal adenocarcinoma cells to grow in the liver of nude rats
}

\author{
ERGÜL EYOL $^{1,4}$, AHMED MURTAGA ${ }^{1}$, MARIA ZHIVKOVA-GALUNSKA ${ }^{1}$, RANIA GEORGES ${ }^{1}$, \\ MICHAEL ZEPP $^{1}$, DOMINIK DJANDJI ${ }^{2}$, JÖRG KLEEFF ${ }^{3}$, MARTIN R. BERGER $^{1}$ and HASSAN ADWAN ${ }^{1}$ \\ ${ }^{1}$ Toxicology and Chemotherapy Unit, German Cancer Research Center, G401, Heidelberg; \\ ${ }^{2}$ German Cancer Research Center, Molecular Immunology, Heidelberg; ${ }^{3}$ Department of General Surgery, \\ Technical University of Munich, Munich, Germany; ${ }^{4}$ Department of Pharmaceutical Toxicology, \\ Faculty of Pharmacy, University of Inonu, 44280 Malatya, Turkey
}

Received June 29, 2012; Accepted August 17, 2012

DOI: $10.3892 / o r .2012 .2049$

\begin{abstract}
Owing to aggressiveness and chemoresistance, pancreatic ductal adenocarcinoma (PDAC) is characterised by a poor prognosis. To address this disease-specific dilemma we aimed to establish animal models, which can be used for identifying new specific tumor markers, as well as serving as tools for potential therapeutic approaches. From a panel of sixteen pancreatic cancer cell lines, two human (Suit2-007 and Suit2-013) and a rat (ASML) cell line were selected for their properties to grow in the liver of male RNU rats and mimic liver metastasis of PDAC. For better monitoring of metastatic tumor growth in vivo, all three pancreatic cancer cell lines were stably transfected with eGFP and luciferase marker genes. In addition, the mRNA expression profile of 13 human PDAC cell lines was analyzed by BeadChip array analysis. Only 33 genes and 5 signaling pathways were identified as significantly associated with the ability of the cell lines to grow initially and/or consistently in rat liver. Only a minority of these genes (osteopontin, matrix metalloproteinase- 1 and insulin-like growth factor 1) has been intensively studied and shown to be closely related to cancer progression. The function of the remaining 30 genes ranges from moderate to poorly investigated, and their function in cancer progression is still unclear. The ensuing three pancreatic cancer liver metastasis models vary in their aggressiveness and macroscopic growth. They will be used for preclinical evaluation of new therapeutic approaches aiming at the genes identified.
\end{abstract}

\section{Introduction}

Pancreatic ductal adenocarcinoma (PDAC) has one of the poorest prognoses of all cancer diseases. For $>90 \%$ of all

Correspondence to: Dr Hassan Adwan, Toxicology and Chemotherapy Unit, Deutsches Krebsforschungszentrum Heidelberg, Im Neuenheimer Feld 581, D-69120 Heidelberg, Germany

E-mail: h.adwan@dkfz.de

Key words: microarray, pancreatic ductal adenocarcinoma cell lines, liver metastasis, animal models diagnosed cases, PDAC ends lethally within the first 6 months. Accordingly, the incidence and mortality of PDAC are almost identical: according to the American Community Survey, there is an average of 10/100,000 new cases/year and PDAC is the fourth leading cause of cancer-related mortality in the Western world (1). Tumor aggressiveness and lack of early symptoms are causal factors for the observation that ca. $80 \%$ of all patients with PDAC have distant metastases at time of diagnosis, which renders the tumor inoperable (2). Furthermore, most patients, who undergo tumor resection, have formed already submicroscopic distant metastases, which become manifest only in the further course of the disease $(3,4)$.

Metastasis is a multistep process that is closely associated with cancer progression. Genetic alterations are crucial for dissemination, migration and invasion of cancer cells, as well as for colonization of a distant organ. Several PDAC-related genes have been identified and characterized as oncogenes or tumor suppressor genes. Despite the better understanding of pancreatic cancer development, the treatment and prognosis of pancreatic cancer have not been substantially improved in the past 30 years. The identification of undiscovered genes as potential tumor markers or drug targets is still one of the most promising ways to increase early diagnosis for this disease and to identify potential novel therapeutic targets. The DNA microarray technology facilitates the simultaneous expression analysis of thousands of genes in relatively short time. However, global gene expression analysis often results in a huge number of genes, for which a causal relationship has to be demonstrated subsequently (5-8). To facilitate this task, we wanted to relate the gene signatures of a panel of PDAC cells to a prominent metastatic property to obtain a relatively short list of genes, which are significantly related to the property chosen.

The impact of new targets can be assessed by suited preclinical models, which simulate tumor formation and metastasis at different stages. For mimicking PDAC, a variety of models has been established. These include xenograft models of human pancreatic cancer cell lines such as MIA PaCa-2, CFPAC-1, Panc-1, Capan-1, Capan-2, BxPC-3, SW1990, AsPC-1, Colo 357 and others (9-6). In addition, rodent cell lines have been used, such as the AS (invasive, but not metastatic) and 
ASML (highly metastatic in vivo, but not invasive) cells, which were derived from the BSp-73 rat pancreatic cancer cell line $(9,17)$. Furthermore, genetic models have become available such as the Mist1-KrasG12D knock-in mouse, which develop mixed differentiation metastatic exocrine pancreatic carcinoma and hepatocellular carcinoma (18).

Orthotopic transplanted models have attracted interest recently. These models are characterized by tumor growth in the organ, from which they have been derived or into which they typically form metastases (12,19-21).

Because orthotopic pancreatic cancer models have rarely been used despite their potential advantages, we wanted to fill into that gap and establish models, which had to meet the following criteria: i) the tumor growth should be easily recordable; ii) the model should mimic liver metastasis, which is the most often affected organ by PDAC; and iii) by using more than one model, the variety of PDAC lesions should be better reflected. Therefore, we transfected human and rat pancreatic cancer cells with appropriate marker genes and selected those, which grew in the rat liver after intra-portal inoculation.

\section{Materials and methods}

Cell culture. All pancreatic cancer cell lines used for transplantation into animals are shown in Table I. The cells were maintained under standard culture conditions at $37^{\circ} \mathrm{C}$ in humidified air with $5 \% \mathrm{CO}_{2}$. For keeping the cells in logarithmic growth, they were propagated 1-3 times per week depending of their growth rate.

Proliferation assay. For determining their proliferation rates, AsML ${ }^{\text {GFP-LUC }}$, Suit2-007 ${ }^{\text {GFP-LUC }}$ and Suit2-013 GFP-LUC cells were seeded at densities of $1 \times 10^{3}, 2 \times 10^{3}, 4 \times 10^{3}, 8 \times 10^{3}$, $16 \times 10^{3}$ cells $/ \mathrm{ml}(100 \mu \mathrm{l} \mathrm{medium} /$ well) into 96 well-plates (flat bottom, Becton-Dickinson, Heidelberg, Germany) and grown for periods of 24, 48, 72 and $96 \mathrm{~h}$. After these periods, $10 \mu \mathrm{l}$ MTT (3-[4.5-dimethylthiazol-2-yl]-2.5-diphenyl tetrazolium bromide, Serva Electrophoresis GmbH, Heidelberg, Germany) solution was added and following an incubation period of $3 \mathrm{~h}$ at $37^{\circ} \mathrm{C}$, the medium was discarded and the cells were lysed by adding $200 \mu \mathrm{l} /$ well acidified 2-propanol $(0.04 \mathrm{~N} \mathrm{HCl})$. After all formazan crystals had been carefully dissolved, the absorption was measured at $540 \mathrm{~nm}$ (reference filter of $690 \mathrm{~nm}$ ) in an automated microtiter plate spectrophotometer (Anthos Mikrosysteme GmbH, Krefeld, Germany). The absorption of exposed cells was given in percent of untreated control cells.

Construction of plasmid and transfection. For obtaining a plasmid containing both, green fluorescent protein (GFP) and luciferase genes, the plasmid pBudCE4.1 was opened with the enzyme BamHI, then blunted and restricted with HindIII (fragment I). In addition, a plasmid containing the luciferase gene was restricted by EcoRI, blunted and restricted again with HindIII (fragment II). Fragments I and II were ligated to obtain a plasmid, which contains the eGFP and luciferase genes (pBudCE4.1 ${ }^{\text {GFP-LUC }}$ ). Subsequently the cell lines ASML, Suit2-013 and S2-007 were transfected with this plasmid.
Transfection with lipofectamine. The ASML, the Suit2-013 and the S2-007 cells were transfected with the plasmid pBudCE4.1 GFP-LUC $^{\text {by using Lipofectamine }}{ }^{\text {TM }}$ Reagent (Invitrogen, Karlsruhe, Germany) as described below. The cells were grown overnight in culture flasks $\left(25 \mathrm{~cm}^{2}\right.$, TPP, Trasadingen, Switzerland) to reach $85-90 \%$ confluence for transfection on the following day. Plasmid DNA $(4 \mu \mathrm{g}$ in $250 \mu \mathrm{l})$ and Lipofectamine $(50 \mu \mathrm{l}$ in $250 \mu \mathrm{l})$ were diluted in Opti-MEM $^{\circledR}$ (Invitrogen) serum-free medium. The mixtures were incubated for $5 \mathrm{~min}$ at room temperature. The diluted DNA was slowly added to the diluted Lipofectamine mix and incubated for $15 \mathrm{~min}$ at room temperature to facilitate the formation of DNA-Lipofectamine (DL) complexes. Corresponding amounts $(500 \mu \mathrm{l} /$ flask $)$ of the DL complexes were added to the cells and incubated at $37^{\circ} \mathrm{C}$ in a $\mathrm{CO}_{2}$ incubator for $4 \mathrm{~h}$. After this period, $50 \%$ of the medium was replaced by fresh culture medium and the cells were further incubated at $37^{\circ} \mathrm{C}$.

Transfection efficiency. After transfection, cells without the plasmid were eliminated by adding the antibiotic zeocin (Invitrogen) to the medium for 72-96 h. Thereafter, green fluorescing cells were positively selected with a FACS-sorter (FACSVantage DiVa, BD Biosciences, Heidelberg, Germany). Transfection efficiency was determined as the number of cells expressing GFP per total number of cells in a given microscopic field and by flow cytometry.

Animals. Male RNU and Wag/Rij rats were used for all in vivo experiments. They were obtained from Charles River (Sulzfeld, Germany) at an age of 5-7 weeks and a corresponding body weight of 120-160 g. They were kept under specific pathogen free (SPF) conditions in Macrolon-III-cages of a ventilated rack (Ventirack, UNO Roestvaststaal B.V., Zevenaar, The Netherlands) providing a 50-fold exchange of filtered air/h as well as positive air pressure inside the cages. Constant room temperature $\left(22 \pm 1^{\circ} \mathrm{C}\right)$, air humidity $(50 \pm 10 \%)$ and dark-lightrhythm $(12 \mathrm{~h})$ were maintained throughout. The animals had free access to autoclaved water and standard laboratory diet. An acclimatization period of 7 days was adhered to before starting any experiments.

Tumor cell transplantation. Logarithmically growing AsML ${ }^{\text {GFP-LUC }}$, Suit2-013 ${ }^{\text {GFP-LUC }}$ and Suit2-007 ${ }^{\text {GFP-LUC }}$ cells were trypsinized and suspended ( $4 \times 10^{6}$ cells) in $0.25 \mathrm{ml}$ PBS (phosphate-buffered saline without calcium and magnesium ions) and $0.15 \mathrm{ml}$ Matrigel (extract of the Engelbreth-Holm-Swarmmouse tumor; Biomatrix EHS solution, Serva Electrophoresis $\mathrm{GmbH})$. This suspension was stored on ice until injection. For tumor cell transplantation, the rats were anaesthetized with isoflurane at $1.5 \mathrm{Vol} \%$ together with $0.5 \mathrm{l} / \mathrm{min}$ oxygen and $1 \mathrm{l} / \mathrm{min}$ nitrous oxide.

After a median laparotomy, the caecum was exteriorized onto a compress moistened with sterile physiological saline and a mesocolic vein was isolated from mesenteric fat. Under microscopic control the tumor cell suspension was injected into this vessel with a 28 -gauge needle.

Thereafter, the vessel was compressed with two cotton swabs for a period of 1-2 min to prevent bleeding; the caecum was moved back into the abdomen; the musculature was 


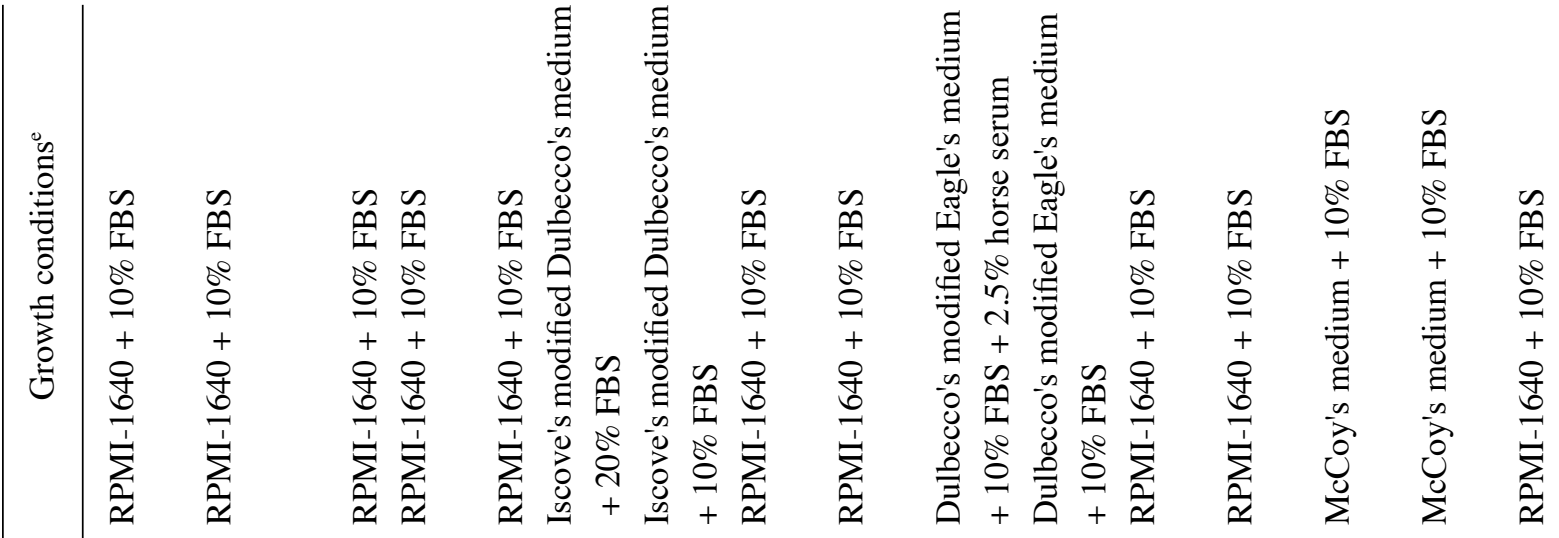

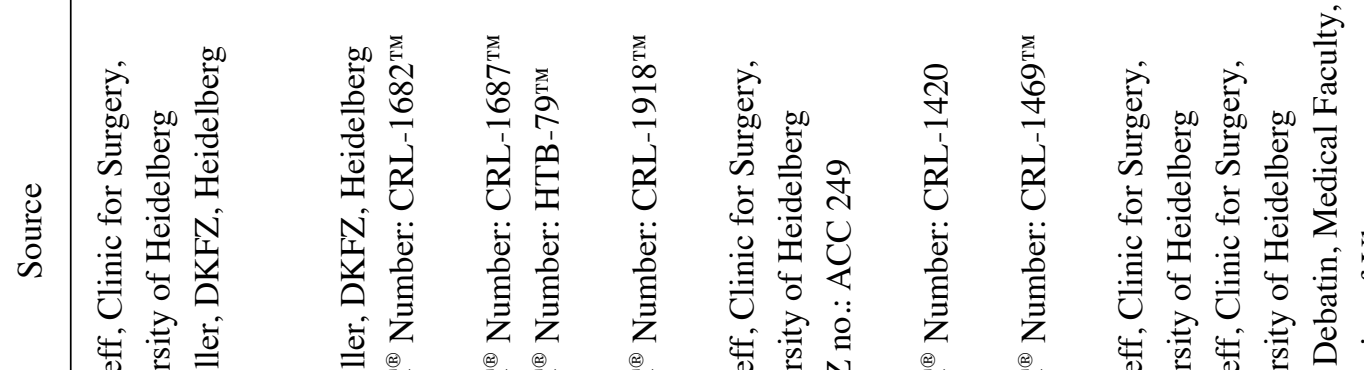

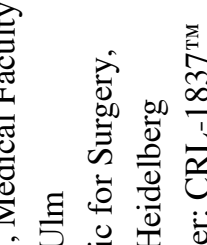

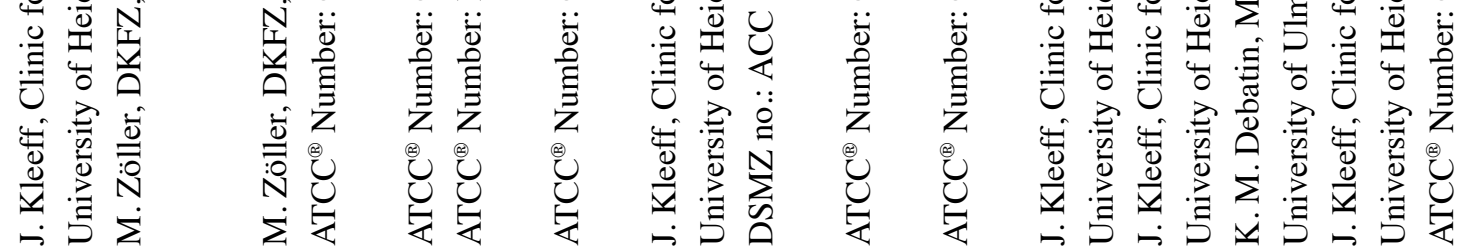

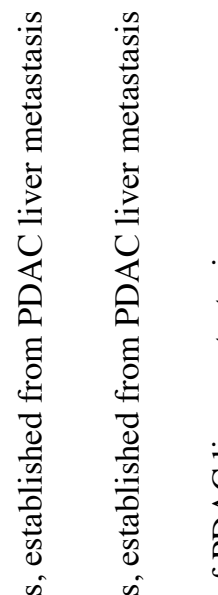

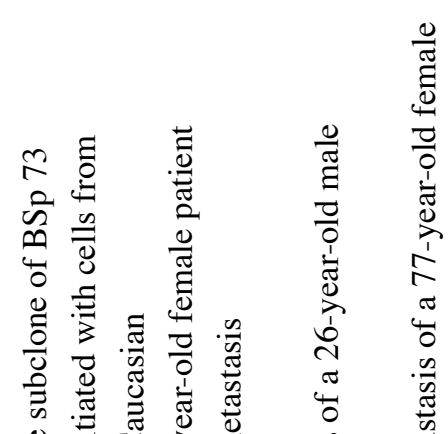

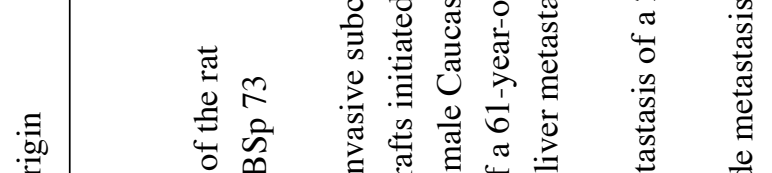

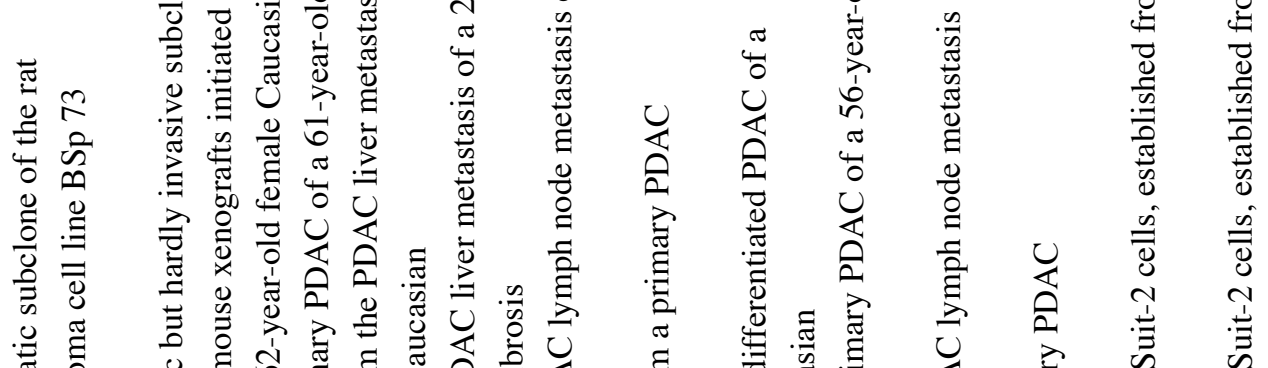

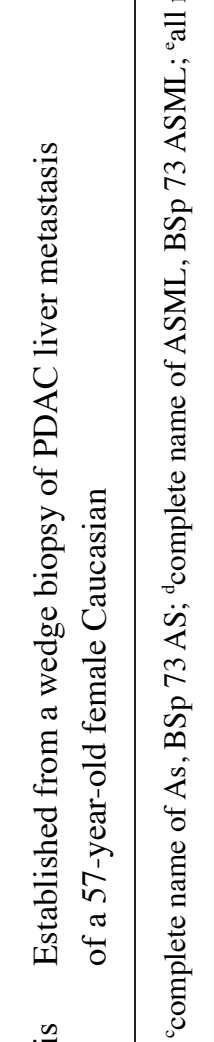

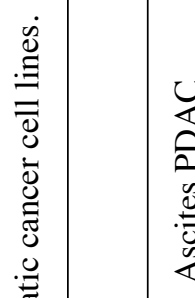

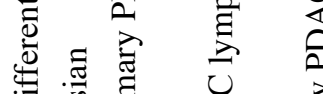

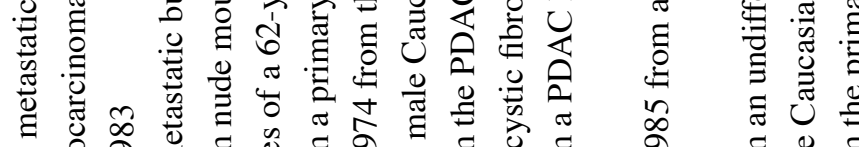

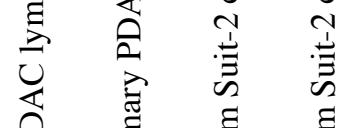

(3)

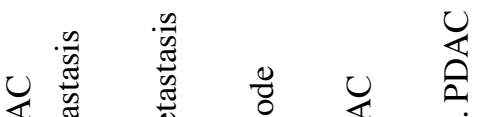

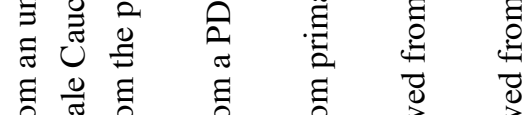

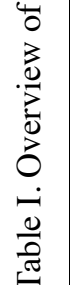

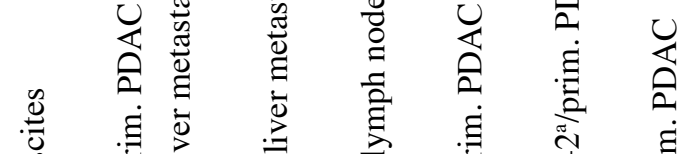

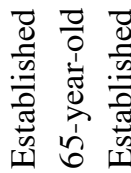

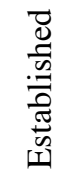

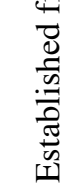

क

$\frac{0}{0}$

$\underset{8}{0}$

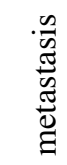

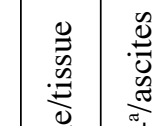

है

焉

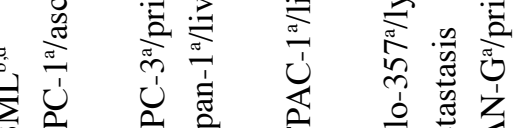

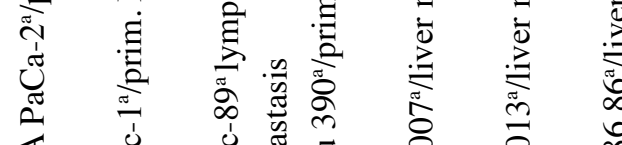

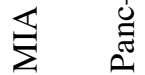

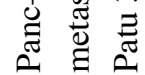


sutured (4-0 vicryl, Ethicon GmbH, Norderstedt, Germany) and the skin closed with metal clips.

Relaparotomy. During this study, all animals were subjected to relaparotomy to monitor the tumor growth in comparison with the bioluminescence imaging (see below). The period until relaparotomy (1-2 weeks) depended on clinical signs of tumor growth as well as on information from earlier passages.

In vivo imaging. Live animal bioluminescence imaging was performed using the IVIS100 imaging system (Xenogen Corp., Alameda, CA, USA). Prior to imaging, the animals were injected intraperitoneally with the substrate D-Luciferin (Synchem Corp., Elk Grove Village, IL, USA) at a dose of $10 \mathrm{mg} / \mathrm{animal}$ and anesthetized with isoflurane/oxygen via the XGI-8 Anesthesia System (Xenogen Corp.), and subsequently analyzed using the Living Image v2.5 software provided by Xenogen Corp. To better identify the location of the signals emitted when imaging live rats, the animals were sacrificed and the liver was removed. Ex vivo imaging of the excised liver was carried out immediately. All animal experiments were performed in accord with the German animal protection law and on the basis of a permit of the respective authority (Regierungspräsidium Karlsruhe).

In vitro imaging. To determine the luciferase activity of AsML ${ }^{\text {GFP-LUC }}$, Suit2-007 $7^{\text {GFP-LUC }}$ and Suit2-013 ${ }^{\text {GFP-LUC }}$ cells, they were plated into 96-well black plates (Nunc Thermo Electron LED GmbH, Langenselbold, Germany) at concentrations of $1 \times 10^{6}, 1 \times 10^{5}, 1 \times 10^{4}, 5 \times 10^{3}$ and $1 \times 10^{3}$ cells/well. Light emission of these cells was monitored using the IVIS-100 imaging system (Xenogen Corp.). As few as 500 cells could be detected in vitro, and yielded a bioluminescent signal ranging from $1 \times 10^{5}$ photons for $2.5 \times 10^{3}$ cells to $4 \times 10^{6}$ photons for $1 \times 10^{6}$ cells.

Animal passages. After the tumor had reached an estimated volume of $1 \mathrm{~cm}^{3}$, the animals were euthanized under anesthesia and the liver was removed under sterile conditions. Tumor cells, which had grown in the liver, were isolated and cultured under normal conditions. After tumor cells had reached confluence, they were used for further portal implantation into rats.

Proliferation rate. An in vitro growth curve was established to determine the mean cell doubling time of the parental and transfected tumor cell lines. The three cell lines showed no difference in cell proliferation with doubling times of $12 \mathrm{~h}$, respectively.

RNA isolation and analysis. Total RNA from 13 PDAC cell lines was isolated using the RNAeasy kit (Qiagen, Hilden, Germany) according to the manufacturer's instructions. RNA was eluted in water. The quality of total RNA was checked by gel analysis using the total RNA Nano chip assay on an Agilent 2100 bioanalyzer (Agilent Technologies GmbH, Berlin, Germany). Only samples with RNA index values $>8.5$ were selected for expression profiling. RNA concentrations were determined using the NanoDrop spectrophotometer (NanoDrop Technologies, Wilmington, DE).

Probe labeling and illumina sentrix BeadChip array hybridization. Biotin-labeled cRNA samples for hybridization on Illumina Human Sentrix-12 BeadChip arrays (Illumina, Inc.) were prepared according to Illumina's recommended sample labeling procedure based on the modified Eberwine protocol (22). In brief, 200 ng total RNA was used for complementary DNA (cDNA) synthesis, followed by an amplification/labeling step (in vitro transcription) to synthesize biotin-labeled cRNA according to the Illumina ${ }^{\circledR}$ Total Prep ${ }^{\mathrm{TM}}$ RNA Amplification kit (Life Technologies). Biotin-16-UTP was purchased from Roche Applied Science (Penzberg, Germany). The cRNA was column purified according to the TotalPrep RNA Amplification kit, and eluted in $80 \mu \mathrm{l}$ of water. Quality of cRNA was controlled using the RNA Nano Chip Assay on an Agilent 2100 bioanalyzer and spectrophotometrically quantified (NanoDrop).

Hybridization was performed at $58^{\circ} \mathrm{C}$, in GEX-HCB buffer (Illumina Inc.) at a concentration of $100 \mathrm{ng}$ cRNA/ $\mu \mathrm{l}$, unsealed in a wet chamber for $20 \mathrm{~h}$. Spike-in controls for low, medium and highly abundant RNAs were added, as well as mismatch control and biotinylation control oligonucleotides. Micro-arrays were washed once in High-Temp Wash buffer (Illumina Inc.) at $55^{\circ} \mathrm{C}$ and then twice in E1BC buffer (Illumina Inc.) at room temperature for $5 \mathrm{~min}$ (and between washed with ethanol at room temperature). After blocking for $5 \mathrm{~min}$ in $4 \mathrm{ml}$ of $1 \%$ (wt/vol) Blocker Casein in phosphatebuffered saline Hammarsten grade (Pierce Biotechnology, Inc., Rockford, IL), array signals were developed by a 10-min incubation in $2 \mathrm{ml}$ of $1 \mu \mathrm{g} / \mathrm{ml} \mathrm{Cy} 3$-streptavidin (Amersham Biosciences, Buckinghamshire, UK) solution and $1 \%$ blocking solution. After a final wash in E1BC, the arrays were dried and scanned.

Scanning and data analysis. Microarray scanning was done using an iScan array scanner. Data extraction was done for all beads individually, and outliers were removed when $>2.5$ MAD (median absolute deviation). All remaining data points were used for the calculation of the mean average signal for a given probe, and standard deviation for each probe was calculated.

Statistical methods. Quantile-normalized Illumina mRNA data were $\log 2$ transformed. Differentially expressed transcripts between cell lines showing growth of tumor cells and those who do not were identified using the empirical Bayes approach (23) based on moderated t-statistics as implemented in the Bioconductor package limma (24). Prior to testing unspecific filtering was applied, selecting transcripts with standard deviation exceeding 0.15 for further analysis. All P-values were adjusted for multiple testing using BenjaminiHochberg correction to control the false discovery rate. In addition to transcript-wise testing, Kyoto Encyclopedia of Genes and Genomes (KEGG) pathway gene sets were tested for regulation between both groups of cell lines using Goeman's global test approach $(25,26)$. Pathway P-values were adjusted for multiple testing using Benjamini-Hochberg correction. All P-values are two-sided. P-values $<0.05$ were 
Table II. Rat organ (compartment) probed for tumour growth (passage 1).

\begin{tabular}{|c|c|c|c|c|}
\hline No. & Cell line & Liver $^{\mathrm{a}, \mathrm{b}}$ & Pancreas ${ }^{a}$ & Peritoneal cavity \\
\hline 1 & A818-4 & $4-0$ & $4-0$ & \\
\hline 2 & AS & $4-4$ & 0 & 0 \\
\hline 3 & $\mathbf{A S M L}^{\mathrm{g}, \mathrm{h}}$ & $>10->10$ & 0 & 0 \\
\hline 4 & AsPC- $1^{\mathrm{d}}$ & $6-3^{c}$ & $4-4^{c}$ & $4-4$ \\
\hline 5 & $\mathrm{BxPC}-3^{\mathrm{e}}$ & $6-2^{c}$ & $2-2^{c}$ & $4-2$ \\
\hline 6 & Capan-1 & $4-0$ & $2-0$ & 0 \\
\hline 7 & CFPAC-1 & $6-2^{c}$ & $2-2$ & \\
\hline 8 & Colo- $357^{\mathrm{f}}$ & $6-2^{c}$ & $2-2^{c}$ & $2-2$ \\
\hline 9 & DAN-G & $4-0$ & $2-0$ & 0 \\
\hline 10 & Mia PaCa-2 & $4-0$ & $2-0$ & 0 \\
\hline 11 & Panc-1 & $4-0$ & $2-0$ & 0 \\
\hline 12 & Panc- $89^{e}$ & $4-1^{\mathrm{c}}$ & $2-0$ & 0 \\
\hline 13 & Patu 390 & $4-0$ & $2-0$ & 0 \\
\hline 14 & $\mathbf{S 2 - 0 0 7 ^ { g , h }}$ & $>10->10$ & 0 & 0 \\
\hline 15 & $\mathbf{S 2 - 0 1 3 ^ { \mathrm { g } , \mathrm { h } }}$ & $>10 ; \sim 80 \%$ & $2-2$ & $2-2$ \\
\hline 16 & Su86.86 & $4-0$ & 0 & 0 \\
\hline
\end{tabular}

${ }^{a}$ Number of rats used-number of rats with tumour growth; baccess to the liver via injection below the liver capsule (I), via intraportal injection (II), and via intrasplenic injection (III). 'Initial growth followed by spontaneous regression. ${ }^{\mathrm{d}}$ Successful growth allowed subsequent passages 1-2 ( $\mathrm{n}=10$ rats); ${ }^{\mathrm{e}}$ successful growth allowed subsequent passages 1-2 ( $\mathrm{n}=12$ rats); ${ }^{\mathrm{f}}$ successful growth allowed subsequent passages 1-4 $\left(\mathrm{n}=24\right.$ rats); ${ }^{\mathrm{g}}$ successful growth allowed subsequent passages $1-5$ ( $\mathrm{n}=40$ rats); ${ }^{\mathrm{h}}$ cell lines in bold were used for the established PDAC rat models (for description see below).

considered statistically significant. All analyses were carried out using R (27).

\section{Results}

In vivo growth of pancreas carcinoma cell lines. Altogether 16 pancreatic carcinoma cell lines were investigated for their potential to form liver metastases (Table II).

Our aim was to identify the most metastatic cell lines. Besides transplantation of tumor cells under the liver capsule or into a mesocolic vein to initiate tumor growth in the liver, alternative routes were tested as well. These included intraperitoneal, intrasplenic and intra-pancreatic injection of cells.

We defined a total take rate of $\geq 50 \%$ of injected rats and stable tumor growth as precondition for further development. Under these conditions, four cell lines qualified, which were ASML, AS, Suit2-013 and Suit2-007 (Table II).

The remaining cell lines showed either a moderate to low take rate, followed by regression of tumor nodules (AsPC-1, BxPC-3, Colo-357 and Panc-89; Table II and Fig. 1) or did not grow in the liver at all (Capan-1, DAN-G, MIA PaCa-2, Panc-1, Patu 390, SU86.86 and T3M4; Table II).

For further characterization of their potential, the cells with insufficient take rate $(<50 \%)$ were implanted into the rat pancreas with the cell line Suit2-013 as positive control. Interestingly, only the cell lines, which showed some growth

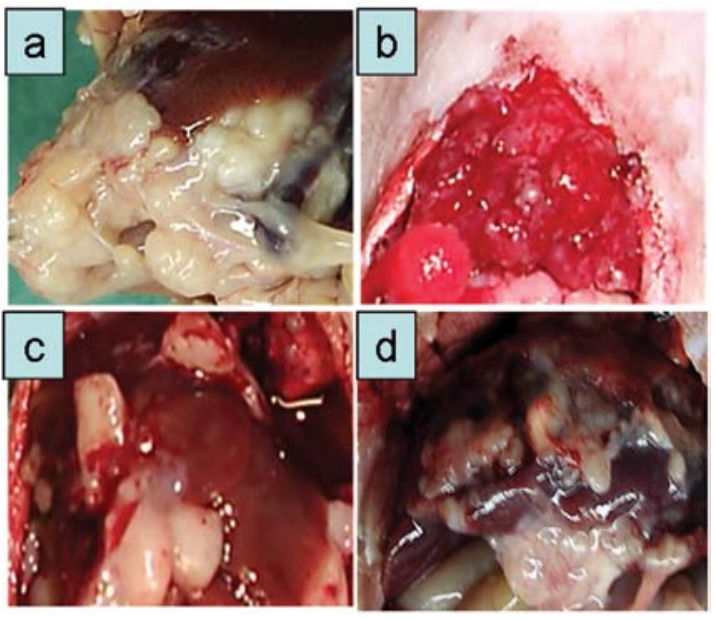

Figure 1. Appearance of metastases one week after intra-portal implantation of pancreatic cancer cells into nude rats. (a) $2 \times 10^{7}$ Colo-357 cells; (b) $2 \times 10^{7}$ AsPC-1 cells; (c) $2 \times 10^{7}$ BxPC- 3 cells; (d) $2 \times 10^{7}$ CFPAC- 1 cells

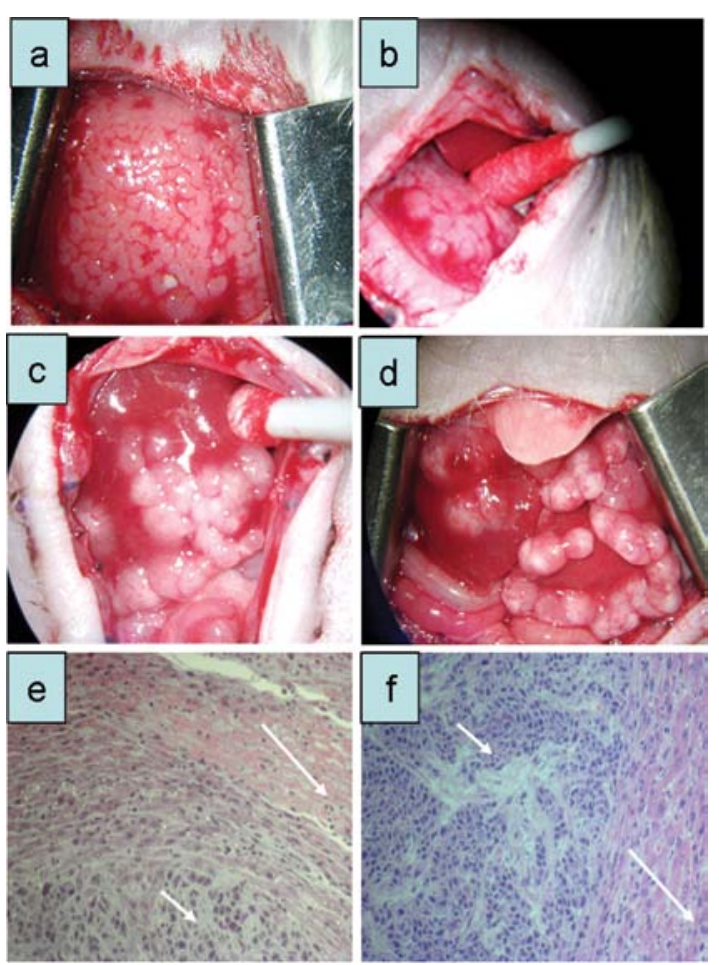

Figure 2. Appearance of metastases one week after intra-portal implantation of ASML ${ }^{\text {GFP-LUC }}$ rat pancreatic cancer cells. (a) $2 \times 10^{7}$ cells in Wag/Rij rats; (b) $2 \times 10^{7}$ cells in RNU nude rats (first passage); (c) $1 \times 10^{7}$ ASML cells in RNU nude rats (third passage); (d) $5 \times 10^{6}$ ASML cells in RNU nude rats (sixth passage); (e) Golden elastica staining of liver tissue after ASML cell inoculation; (f) haematoxylin and eosin staining of liver tissue after ASML cell inoculation.

in the liver, grew also in the rat pancreas. Finally, these cell lines showed also tumor spread after implantation into the peritoneal cavity, except for Panc-89 cells.

ASML model. ASML cells are derived from a pancreas adenocarcinoma growing in BDX rats. Since this strain is not commercially available, another strain of immunocompetent rats (Wag/Rij) was used, instead. Wag/Rij rats 


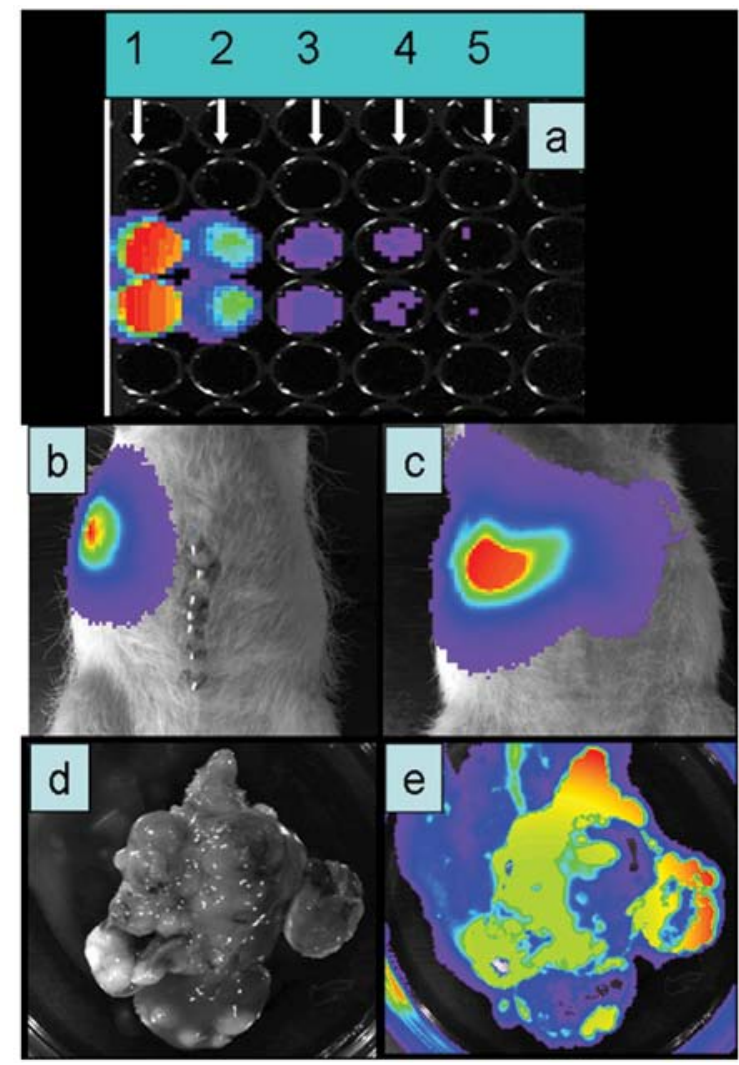

Figure 3. Light emission based on luciferase activity of ASML GFP-LUC rat pancreatic cancer cells. (a) Light emission of ASML ${ }^{\text {GFP-LUC }}$ cells in vitro. 1) $1 \times 10^{6}$ cells; 2) $1 \times 10^{5}$ cells; 3) $1 \times 10^{4}$ cells; 4) $5 \times 10^{3}$ cells; 5) $2.5 \times 10^{3}$ cells. (b) Aspect of tumor bearing animal 14 days following transplantation of ASML ${ }^{\text {GPP-LUC }}$ cells. (c) Aspect after luciferin injection of tumor bearing animal 21 days following transplantation of ASML ${ }^{\text {GFP-LUC }}$ cells. (d) Liver of this rat before luciferin exposure. (e) Light emission of this liver after luciferin exposure.

implanted with ASML ${ }^{\text {GFP-LUC }}$ cells showed clearly visible tumor growth after the first week, but complete remission of all tumor masses after two weeks. Therefore, nude rats (RNU-Strain) were used for all subsequent experiments. In immuno-compromised rats, $2 \times 10^{7} \mathrm{ASML}^{\text {GFP-LUC }}$ cells caused steady tumor growth in passages 1-4. Thereafter the tumor cell number was slowly reduced to $5 \times 10^{6}$ cells in the sixth passage (Fig. 2b-d). With increasing number of tumor passages a distinct macroscopic picture emerged (Fig. 2b-d). Tumor nodules appeared at the liver lobe edges, showing a pearl-necklace like growth (Fig. 2d). The corresponding histological pictures are shown in Fig. 2e and f. The carcinoma showed nodular growth with large amounts of fibrous connective tissue. Tumor nodules were clearly separated from the normal liver tissue.

ASML cells from passage 6 were transfected with the plasmid pBudCE4.1 ${ }^{\text {GFP-LUC }}$ containing the GFP and luciferase genes under the control of the promoter CMV. In vitro, following exposure to luciferin, a light signal was detectable down to $5 \times 10^{3}$ cells (Fig. 3a). Two weeks following transplantation of ASML ${ }^{\text {GFP-LUC }}$ cells a clear light signal was obtained after injection of luciferin (10 mg/animal; Fig. $3 \mathrm{~b}$ and c). After liver explanation, it was confirmed that the light emission correlated very well with the presence of tumor nodules (Fig. 3d and e).

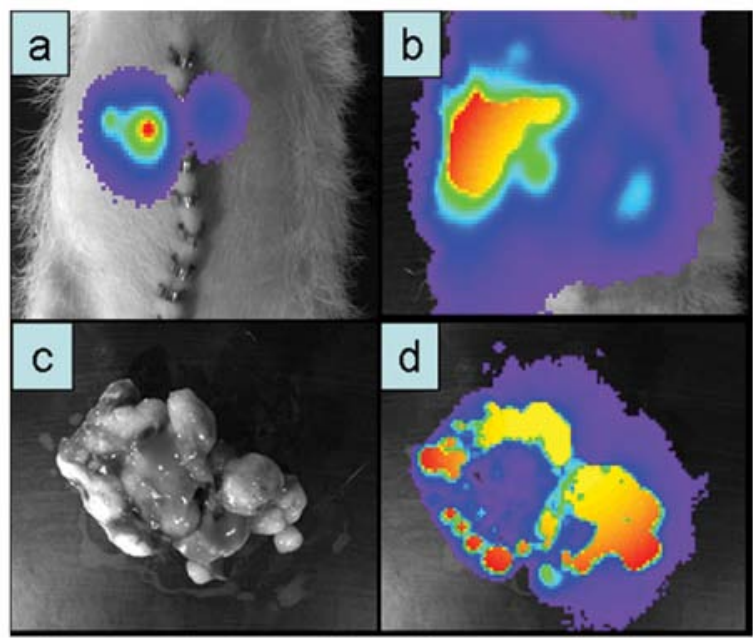

Figure 4. Appearance of metastases 2-3 weeks after intra-portal implantation of Suit2-007 $7^{\text {GFP-LUC }}$ human pancreatic cancer cells. (a) Aspect of a tumor bearing animal after luciferin injection 14 days following transplantation of $1 \times 10^{7}$ Suit2-007 $7^{\text {GFP-LUC }}$ cells. (b) Aspect of this animal after 21 days following transplantation. (c) Liver of this rat before luciferin exposure. (d) Light emission of this liver after luciferin exposure.
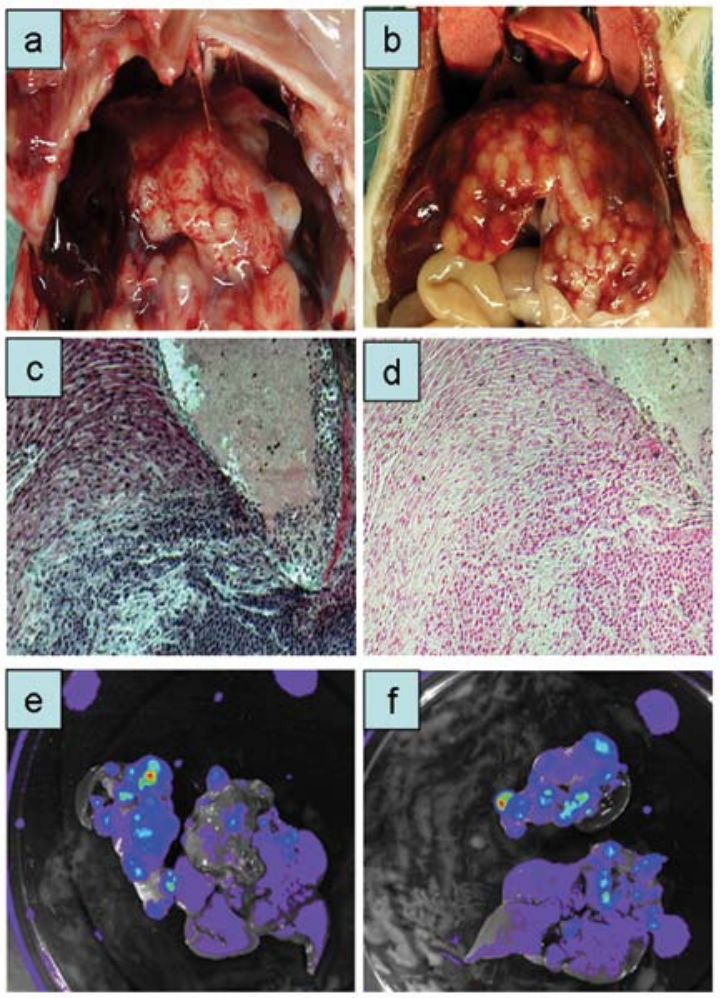

Figure 5. Metastases formation of Suit-2013 ${ }^{\text {GFP-LUC }}$ human pancreatic cancer cells after implantation into the liver of nude rats. (a) $1 \times 10^{7}$ Suit2-013 $3^{\text {GPP-LUC }}$ cells implanted under liver capsule (first passage). (b) $5 \times 10^{6}$ Suit2-013 ${ }^{\text {GFP-LUC }}$ cells implanted intra-portally, with irradiation (third passage). (c) Golden elastica staining of liver tissue. (d) Iron staining of liver tissue. (e) Ex vivo detection of GFP in the liver shown in (c and d). (f) Ex vivo detection of luciferase activity in the liver shown in (c and d).

Suit2-007 model. Suit-2 cells were originally isolated from a 73-year-old Japanese man, who had developed a metastatic pancreatic carcinoma in the liver. From this mother cell line, 
Table III. Grouping of human PDAC cell lines according to their growth behavior in the liver of nude rats.

\begin{tabular}{|c|c|c|c|c|c|c|c|}
\hline Cell lines & Growth in rat liver/category & G1 & $\mathrm{G} 2$ & G3 & G4 & G5 & G6 \\
\hline Capan-1 & $-/ \mathrm{C} 1$ & - & - & - & - & NV & - \\
\hline DAN-G & $-/ \mathrm{C} 1$ & - & - & - & - & NV & - \\
\hline MIA PaCa-2 & $-/ \mathrm{C} 1$ & - & - & - & - & NV & - \\
\hline Panc-1 & $-/ C 1$ & - & - & - & - & NV & - \\
\hline Patu 390 & $-/ \mathrm{C} 1$ & - & - & - & - & NV & - \\
\hline SU86.86 & $-/ \mathrm{C} 1$ & - & - & - & - & NV & - \\
\hline CFPAC-1 & $+? / \mathrm{C} 2$ & - & - & - & - & NV & $+?$ \\
\hline NG AsPC-1 & $+? / \mathrm{C} 2$ & - & + & - & - & $\mathrm{NV}$ & $+?$ \\
\hline Panc-89 & $+? / \mathrm{C} 2$ & - & + & - & - & NV & $+?$ \\
\hline BxPC-3 & $++/ \mathrm{C} 3$ & + & + & - & $\mathrm{NV}$ & - & ++ \\
\hline Colo-357 & $++/ \mathrm{C} 3$ & + & - & - & $\mathrm{NV}$ & - & ++ \\
\hline S2-007 & $+++/ C 4$ & + & + & + & + & + & +++ \\
\hline S2-013 & $+++/ \mathrm{C} 4$ & + & + & + & + & + & +++ \\
\hline
\end{tabular}

$\mathrm{C} 1$, category 1 contains cell lines, which did not grow in the liver of nude rats (-). C2, category 2 contains cell lines which grew poorly and regressed within 2 weeks (+?). C3, category 3 contains cell lines, which initially grew distinctly in the liver of nude rats, but regressed thereafter $(++)$. C4, category 4 contains cell lines which grew consistently in the liver of nude rats (+++). G1, group 1, contains cell lines which initially grew distinctly (with or without spontaneous regression thereafter) in the liver of nude rats (+) vs. the remaining cell lines (-). G2, group 2, contains cell lines which initially grew distinctly or poorly (with or without spontaneous regression thereafter) in the liver of nude rats $(+)$ vs. the remaining cell lines (-). G3, group 3, contains cell lines which grew consistently in the liver of nude rats (+), vs. the remaining cell lines (-). G4, group 4, contains cell lines which grew consistently in the liver of nude rats (+), vs. the remaining cell lines (-), with cell lines showing initial growth being excluded. G5, group 5, contains cell lines which grew consistently in the liver of nude rats (+), vs. cell lines, which initially grew distinctly and regressed thereafter (-), with the remaining cell lines being excluded. G6, group 6, contains cell lines which were ordered according to their increasing ability to grow in the liver of nude rats and tested pairwise for altered gene expression. NV, cell lines excluded from the respective comparison.

28 sub-clones were originally established by animal passage (28). The S2-007 cell line is a sub-clone of Suit-2 cells, which was found very aggressive and able to metastasize into the liver $(28,29)$.

Suit2-007 cells/animal $\left(1 \times 10^{7}\right)$ were injected intra-portally into nude rats. Two weeks post-injection liver tumors could be detected. Tumor cells, which had grown in the liver were then isolated and cultured for the next animal passage. This procedure was repeated three times. After the last passage, a new Suit2-007 sub-clone was established that was consistently able to form liver metastasis in nude rats, with nodules appearing one week after implantation. Similar to the ASML cells, this sub-clone was thereafter stably transfected with the plasmid pBudCE4.1 $1^{\text {GFP-LUC }}$ (Fig. 4).

S2-013 model. Suit2-013 is another sub-clone of Suit-2, which was used for establishing a less aggressively growing nude rat model. After the first intra-portal injection, the cells grew moderately in the liver and spontaneously regressed in some of the animals.

Following GFP and luciferase transfection, $2 \times 10^{6}$ S2-013 ${ }^{\text {GFP-LUC }}$ cells were implanted under the capsule of the middle liver lobe of a nude rat. After 24 days, stable tumor growth was observed (passage 1; Fig. 5a). Subsequently, $1 \times 10^{7}$ cells were injected intra-portally and caused visible tumor growth in implanted rats after 3-4 weeks (Fig. 5b), which partly showed spontaneous regression.

To facilitate the growth of this cell line, two nude rats received total body irradiation (5 Gy) three days before tumor cell implantation. As expected, the period until visible tumor growth occurred was shorter in these rats (19 days). One of the two irradiated animals received intra-pancreatic tumor implantation in addition to the intra-portal implantation. Upon histological examination, the S2-013 ${ }^{\text {GFP-LUC }}$ cells showed infiltrating growth into normal liver tissue and production of fibrous connective tissue (Fig. 5c and d).

When pancreas and liver of this animal were excised at autopsy, both organs were loaded with GFP and luciferasepositive cells upon ex vivo detection (Fig. 5e and f).

In vivo imaging. One week after tumor cell implantation, animals were analyzed by non-invasive bioluminescent imaging. After intra-peritoneal administration of D-luciferin, the rats were examined for bioluminescent signals originating from tumor cells growing in the liver. A clear light signal $\left(1 \times 10^{5}\right.$ photons/sec) could be detected from the liver region of the respective animals. To confirm this result, the affected organs were excised and imaged again. Although not all nodules yielded a luciferase positive signal, the obtained signals were emitted only from the liver. 
Table IV. Overview of genes with significantly altered expression.

\begin{tabular}{|c|c|c|c|c|c|c|c|c|}
\hline Gene & G1 & $\mathrm{G} 2$ & G3 & G4 & G5 & G6 & Function $^{\mathrm{a}}$ & Relation to cancer ${ }^{\mathrm{a}, \mathrm{b}}$ \\
\hline SPP1 (OPN) & + & + & & + & & + & +++++ & +++++ \\
\hline UPK1B & + & + & + & + & & & + & $(+)$ \\
\hline FXYD2 & & & + & + & & & + & - \\
\hline SLC1A1 & & & + & + & & + & ++ & + \\
\hline EDAR & & & + & + & & + & + & $(+)$ \\
\hline IGF2 & & & + & + & & & ++++ & +++ \\
\hline FOXL2 & & & + & & & & +++ & + \\
\hline C3orf72 & & & + & & & & - & - \\
\hline DPEP1 & & & + & & & & + & + \\
\hline HSPA12A & & & + & + & & & + & - \\
\hline VNN2 & & & + & & & & + & - \\
\hline ELA3A & & & + & & & & - & - \\
\hline R3HDML & & & + & & & & - & - \\
\hline MMP-1 & & & + & & & & ++++ & +++ \\
\hline FAM150A & & & + & & & & - & - \\
\hline ARSE & & & + & & & & + & - \\
\hline AGFG2 (HRBL) & & & + & & & & + & - \\
\hline HSD17B2 & & & + & & & & + & + \\
\hline HDHD1A & & & + & & & & $(+)$ & - \\
\hline NCKAP5 & & & + & & & & + & - \\
\hline LOC642342 & & & + & & & & - & - \\
\hline CISD2 & & & + & & & & + & - \\
\hline BCL2L11 & & & + & & & & + & + \\
\hline SLITRK4 & & & + & & & & $(+)$ & $(+)$ \\
\hline UBASH3B & & & + & & & & + & - \\
\hline KITLG & & & + & & & & ++ & + \\
\hline TGFBR3 & & & + & & & & ++ & + \\
\hline LOC642975 & & & + & & & & - & - \\
\hline ADNP2 & & & + & & & & + & - \\
\hline NR5A2 (LRH-1) & & & + & & & & +++ & +++ \\
\hline TMSB15A & & & + & & & & $(+)$ & $(+)$ \\
\hline CA12 & & & & + & & & + & + \\
\hline BASP1 & & & & & + & & + & $(+)$ \\
\hline TM4SF18 & & & & & + & & $(+)$ & $(+)$ \\
\hline
\end{tabular}

${ }^{a, b}$ Number of citations listed in PubMed (15.03.2012) for the respective genes. The PubMed citations were grouped according to the overall description of the gene ${ }^{\mathrm{a}}$ or its relation to cancer ${ }^{\mathrm{b}}$.,$+++++ \geq 1000 ;++++, \geq 500 ;+++, \geq 200 ;++, \geq 100 ;+, 3-100 ;(+), 1-3$ and -, no publication listed.

mRNA expression profile of 13 human PDAC cell lines. The mRNA expression profile of 13 human PDAC cell lines was analyzed by Illumina Human Sentrix-12 BeadChip arrays. For comparison, the cell lines were grouped according to their growth behavior, into 4 categories (Table III).

Category 1 comprised those 6 cell lines, which did not grow in the liver of nude rats. Category 2 comprised the 3 cell lines, which grew poorly and regressed within 2 weeks.

Category 3 included the 2 cell lines, which initially grew distinctly in the liver of nude rats, but nevertheless regressed thereafter.
Category 4 comprised the 2 cell lines, which grew consistently in the liver of nude rats.

For a comprehensive analysis, these categories were grouped in different ways as shown in Table III. For identifying genes, which are involved in the early growth of PDAC cells in rat liver, we grouped categories 2, 3 and 4 (group 1), as well as categories 3 and 4 (group 2) and compared them with the remaining cell lines (Table III). Remarkably, only 2 genes (OPN and UTP14A) showed significantly changed expression ( $P>0.05)$ in growing cell lines, as compared to those, which did not. Subsequently, for identifying genes, which are crucial 
for consistent growth in the liver of nude rats, we compared category 4 vs. all other cell lines (categories 1, 2 and 3, group 3, Table III). In addition, category 4 was compared with categories 1 and 2 to exclude those cell lines from the remaining panel, which initially grew distinctly in the liver of nude rats, but regressed thereafter (group 4). Furthermore, category 4 was compared with category 3 (group 5). Interestingly, the number of differentially expressed genes was higher than in the first approach: 30, 8 and 2 genes showed significantly altered expression when analyzing groups 3,4 and 5, respectively (Table IV).

Finally, all 4 categories were used to search for incremental differences in mRNA expression (group 6). From this comparison, 3 genes emerged as being significantly modulated in a progression dependent way. These genes were OPN, SLC1A1 and EDAR (Table IV).

Signaling pathways involved in the metastatic process. Two hundred and thirty signaling pathways (PW) involved in cellular growth were analyzed in PDAC cell lines for their relation to the cell line growth in the liver of nude rats. This analysis indicated that 5 PWs were significantly and 3 more were likely involved in this process when analyzing groups 3 , 4 and 5. There were no signaling PWs significantly involved, when analyzing the other groups.

\section{Discussion}

Research on pancreatic cancer has been aiming to improve the understanding of the sublying molecular characteristics and to develop new treatment options for this malignant disease. However, due to the failure of current antineoplastic drugs, metastatic PDAC is still one of the most lethal cancers $(2,4,30)$.

In this study, we intended to establish representative and reproducible in vivo models that mimic metastasis formation of pancreatic cancer in the liver. In addition, we wanted to identify those genes, which are instrumental for the metastatic process. Therefore, we determined whether the PDAC cell lines would have the property to grow in rat liver. Subsequently, we used this capability as readout for identifying genes, which differ in their expression level between PDAC cells that grew in rat liver and those that did not. Remarkably, this comparison resulted in a group of only 33 genes, which were significantly associated with the ability of the cell lines to grow in rat liver initially and/or consistently.

These genes can be grouped into three subgroups. First, there were genes, which are well known for their role in cancer progression such as OPN, MMP-1 and IGF2 (31-35). Based on the categories of metastatic growth and our inter-group comparisons, OPN seems to be required for the initial growth of PDAC cells in rat liver, while MMP-1 and EGF1 are more important for their permanent growth.

The next group contained 5 genes, which are less well characterized and reportedly have anti-invasive properties such as DPEP1, or contribute to cancer progression such as SLC1A1, C3orf72 and KITLG.

Finally, the largest group of 25 genes has not (yet) been associated with cancer.

To detect functional relationships, the affiliation of these genes to known signaling pathways was analyzed.
Surprisingly, the significantly altered genes were firmly associated to only $2 \%$ (5 of 230) of all pathways. Two of these associated signaling pathways are the PPAR and the bladder cancer signaling pathways, which have been implicated in a variety of neoplastic processes, such as breast, bladder and colon cancer (36-38). Another related pathway is the 'Maturity Onset Diabetes' pathway. A possible link between diabetes and PDAC has emerged recently. A meta-analysis of a total of 36 studies (17 case-control and 19 cohort or nested casecontrol studies) demonstrated that patients with diabetes type 2 have a 1.8 higher risk to develop PDAC than people without diabetes (40).

The remaining two pathways 'Proximal tubule bicarbonate reclamation' and 'Aldosterone-regulated sodium reabsorption' pathways have not yet been found to be implicated in neoplastic processes.

The detected low number of genes affiliated to growth of PDAC cell lines in rat liver is astonishing and might be due to various reasons. Our approach to select the expression of the whole genome of 13 cell lines resulted in a large number of comparisons, which in turn allowed detecting only those genes with most significantly altered expression. Therefore, a relatively large number of falsely negative genes cannot be excluded. Also, using the in vitro signature of PDAC cell expression might be responsible for missing another category of genes. These are genes, which differ in expression between in vitro and in vivo conditions. Recently, examples have been described for genes, which show a strong modulation of their expression upon transplantation into rat liver (40-42).

It is hoped that a future analysis of these gene functions and their respective signaling cascades will contribute to better understand why only two human cancer cell lines were able to grow consistently in the liver of nude rats. In the absence of such an analysis, the established tumor models will allow investigating the course of metastasis formation in pancreatic cancer and can be used for examining new therapeutics.

The models were based on transplanting PDAC cells into the liver via the intra-portal route, as previously described (43). This type of injection mimics to a certain extent the natural way of pancreatic cancer cell dissemination into the liver and can be used to investigate the efficacy of new antineoplastic drugs against PDAC cells invading and colonizing the liver.

In general, metastasis formation follows two principles. According to the mechanical principle, which was described by Ewing, the tumor cells are caught in the blood capillaries of their target organs $(44,45)$. Alternatively, the seed and soil principle of Paget describes the growth of tumor cells in organs, which offer appropriate growth conditions (46).

The investigation of genes identified in this study by targeted knock down may help to learn/discriminate, which of the two principles has greater influence on PDAC cell colonization of rat liver.

Recording of tumor growth inside experimental animals requires appropriate methods for detection. Therefore, we stably transfected the tumor cells with luciferase and GFP as markers, respectively. One problem with the transfection of genetic markers is, that a sub-clone may express new proteins, which can be recognized by the immune system. 
Then, transfected cells can lose their metastatic ability or change their growth behavior in vivo, as reported after transfection with EGFP and Lac Z- markers, respectively $(43,47)$. Indeed, this phenomenon was recognized in Suit2$013^{\text {GFP-LUC }}$ and partially in Suit2-007 $7^{\text {GFP-LUC }}$ cells. After transfection of Suit2-013 cells with GFP and luciferase, the generated Suit2-013 ${ }^{\text {GFP-LUC }}$ cells grew slowly and had a take rate of less than $50 \%$. Only after pre-irradiation of the animals, these cells grew more efficiently.

In summary, 3 of 16 PDAC cell lines were able to grow consistently in the liver of male RNU rats and served as basis for three newly established orthotopic PDAC liver metastasis xenograft models in male RNU rats. Based on the ability of 13 human PDAC cell lines to grow initially and consistently in the liver of nude rats, and the genetic signature of these cell lines as determined by microarray analysis, 33 genes were identified to be significantly altered as compared to the expression signature of cells without this capability. Only a minority of these genes (OPN, MMP-1 and IGF2) has been investigated thoroughly and shown to be involved in cancer progression. The vast majority of these genes have not yet been sufficiently evaluated and their function in cancer progression is unclear. However, further studies should clarify their function in and relationship to cancer.

\section{References}

1. Jemal A, Tiwari RC, Murray T, et al: Cancer statistics, 2004. CA Cancer J Clin 54: 8-29, 2004.

2. Ghaneh P, Kawesha A, Howes N, Jones L and Neoptolemos JP: Adjuvant therapy for pancreatic cancer. World J Surg 23: 937-945, 1999.

3. Alanen KA and Joensuu H: Long-term survival after pancreatic adenocarcinoma - often a misdiagnosis? Br J Cancer 68 1004-1005, 1993.

4. Li D, Xie K, Wolff R and Abbruzzese JL: Pancreatic cancer. Lancet 363: 1049-1057, 2004.

5. Friess H, Ding J, Kleeff J, et al: Microarray-based identification of differentially expressed growth- and metastasis-associated genes in pancreatic cancer. Cell Mol Life Sci 60: 1180-1199, 2003.

6. Kayed H, Kleeff J, Keleg S, et al: Indian hedgehog signaling pathway: expression and regulation in pancreatic cancer. Int $\mathrm{J}$ Cancer 110: 668-676, 2004

7. Bardeesy $\mathrm{N}$ and DePinho RA: Pancreatic cancer biology and genetics. Nat Rev Cancer 2: 897-909, 2002.

8. Hansel DE, Kern SE and Hruban RH: Molecular pathogenesis of pancreatic cancer. Annu Rev Genomics Hum Genet 4: 237-256, 2003.

9. Matzku S, Komitowski D, Mildenberger M and Zoller M: Characterization of BSp73, a spontaneous rat tumor and its in vivo selected variants showing different metastasizing capacities. Invasion Metastasis 3: 109-123, 1983.

10. Katz MH, Takimoto S, Spivack D, Moossa AR, Hoffman RM and Bouvet M: A novel red fluorescent protein orthotopic pancreatic cancer model for the preclinical evaluation of chemotherapeutics. J Surg Res 113: 151-160, 2003.

11. Sun FX, Tohgo A, Bouvet M, Yagi S, Nassirpour R, Moossa AR and Hoffman RM: Efficacy of camptothecin analog DX-8951f (Exatecan Mesylate) on human pancreatic cancer in an orthotopic metastatic model. Cancer Res 63: 80-85, 2003.

12. Marincola FM, Drucker BJ, Siao DY, Hough KL and Holder Jr WD: The nude mouse as a model for the study of human pancreatic cancer. J Surg Res 47: 520-529, 1989.

13. Jimenez RE, Hartwig W, Antoniu BA, Compton CC, Warshaw AL and Fernandez-Del CC: Effect of matrix metalloproteinase inhibition on pancreatic cancer invasion and metastasis: an additive strategy for cancer control. Ann Surg 231: 644-654, 2000.
14. Tzanakakis GN, Margioris AN, Tsatsakis AM and Vezeridis MP: The metastatic potential of human pancreatic cell lines in the liver of nude mice correlates well with cathepsin B activity. Int J Gastrointest Cancer 34: 27-38, 2003.

15. Vernejoul F, Faure P, Benali N, et al: Antitumor effect of in vivo somatostatin receptor subtype 2 gene transfer in primary and metastatic pancreatic cancer models. Cancer Res 62: 6124-6131, 2002.

16. Samnick S, Romeike BF, Kubuschok B, et al: p-[123I]iodoL-phenylalanine for detection of pancreatic cancer: basic investigations of the uptake characteristics in primary human pancreatic tumour cells and evaluation in in vivo models of human pancreatic adenocarcinoma. Eur J Nucl Med Mol Imaging 31: 532-541, 2004.

17. Raz A, Zoller M and Ben Z: Cell configuration and adhesive properties of metastasizing and non-metastasizing BSp73 rat adenocarcinoma cells. Exp Cell Res 162: 127-141, 1986.

18. Tuveson DA, Zhu L, Gopinathan A, et al: Mist1-KrasG12D knock-in mice develop mixed differentiation metastatic exocrine pancreatic carcinoma and hepatocellular carcinoma. Cancer Res 66: 242-247, 2006.

19. Kokkinakis DM, Ahmed MM, Chendil D, Moschel RC and Pegg AE: Sensitization of pancreatic tumor xenografts to carmustine and temozolomide by inactivation of their O6-Methylguanine-DNA methyltransferase with O6-benzylguanine or O6-benzyl-2'-deoxyguanosine. Clin Cancer Res 9: 3801-3807, 2003.

20. Ito D, Fujimoto K, Mori T, et al: In vivo antitumor effect of the mTOR inhibitor CCI-779 and gemcitabine in xenograft models of human pancreatic cancer. Int J Cancer 118: 2337-2343, 2006.

21. Sausville EA and Burger AM: Contributions of human tumor xenografts to anticancer drug development. Cancer Res 66: 3351-3354, 2006.

22. Eberwine J, Yeh H, Miyashiro K, et al: Analysis of gene expression in single live neurons. Proc Natl Acad Sci USA 89: 3010-3014, 1992.

23. Smyth GK: Linear models and empirical bayes methods for assessing differential expression in microarray experiments. Stat Appl Genet Mol Biol 3: Article3, 2004.

24. Smyth GK: Limma: linear models for microarray data. In: Bioinformatics and Computational Biology Solutions Using R and Bioconductor. Gentleman R, Carey V, Dudoit S, Irizarry R, and Huber W (eds). Springer, New York, pp397-420, 2005.

25. Goeman JJ, van de Geer SA, de KF and van Houwelingen HC: A global test for groups of genes: testing association with a clinical outcome. Bioinformatics 20: 93-99, 2004.

26. Goeman JJ, Oosting J, Cleton-Jansen AM, Anninga JK and van Houwelingen HC: Testing association of a pathway with survival using gene expression data. Bioinformatics 21 : 1950-1957, 2005.

27. $\mathrm{R}$ Development Core Team: A language and environment for statistical computing. R Foundation for Statistical Computing. Vienna, 2011.

28. Iwamura T, Katsuki T and Ide K: Establishment and characterization of a human pancreatic cancer cell line (SUIT-2) producing carcinoembryonic antigen and carbohydrate antigen 19-9. Jpn J Cancer Res 78: 54-62, 1987.

29. Taniguchi S, Iwamura T and Katsuki T: Correlation between spontaneous metastatic potential and type I collagenolytic activity in a human pancreatic cancer cell line (SUIT-2) and sublines. Clin Exp Metastasis 10: 259-266, 1992.

30. Friess H, Buchler M, Kruger M and Beger HG: Treatment of duct carcinoma of the pancreas with the LH-RH analogue buserelin. Pancreas 7: 516-521, 1992.

31. Fedarko NS, Jain A, Karadag A, Van Eman MR and Fisher LW: Elevated serum bone sialoprotein and osteopontin in colon, breast, prostate, and lung cancer. Clin Cancer Res 7: 4060-4066, 2001.

32. Kolb A, Kleeff J, Guweidhi A, et al: Osteopontin influences the invasiveness of pancreatic cancer cells and is increased in neoplastic and inflammatory conditions. Cancer Biol Ther 4: 740-746, 2005.

33. Wong JC, Chan SK, Schaeffer DF, et al: Absence of MMP2 expression correlates with poor clinical outcomes in rectal cancer, and is distinct from MMP1-related outcomes in colon cancer. Clin Cancer Res 17: 4167-4176, 2011.

34. Hart K, Landvik NE, Lind H, Skaug V, Haugen A and Zienolddiny S: A combination of functional polymorphisms in the CASP8, MMP1, IL10 and SEPS1 genes affects risk of non-small cell lung cancer. Lung Cancer 71: 123-129, 2011. 
35. Hoyo C, Murphy SK, Schildkraut JM, et al: IGF2R genetic variants, circulating IGF2 concentrations and colon cancer risk in African Americans and Whites. Dis Markers 32: 133-141, 2012.

36. Yee LD, Sabourin CL, Liu L, Li HM, Smith PJ, Seewaldt V and Kniss DA: Peroxisome proliferator-activated receptor gamma activation in human breast cancer. Int J Oncol 15: 967-973, 1999.

37. Guan YF, Zhang YH, Breyer RM, Davis L and Breyer MD: Expression of peroxisome proliferator-activated receptor gamma (PPARgamma) in human transitional bladder cancer and its role in inducing cell death. Neoplasia 1: 330-339, 1999.

38. Gupta RA, Brockman JA, Sarraf P, Willson TM and DuBois RN: Target genes of peroxisome proliferator-activated receptor gamma in colorectal cancer cells. J Biol Chem 276: 29681-29687, 2001.

39. Huxley R, Ansary-Moghaddam A, Berrington de GA, Barzi $\mathrm{F}$ and Woodward M: Type-II diabetes and pancreatic cancer: a meta-analysis of 36 studies. Br J Cancer 92: 20762083, 2005.

40. Uhlmann ME, Georges RB, Boleij A, Eyol E, Kubarenko A, Adwan $\mathrm{H}$ and Berger MR: Influence of osteopontin expression on the metastatic growth of CC531 rat colorectal carcinoma cells in rat liver. Cancer Gene Ther 18: 795-805, 2011.
41. Georges RB, Adwan H, Hamdi H, Hielscher T, Linnemann U and Berger MR: The insulin-like growth factor binding proteins 3 and 7 are associated with colorectal cancer and liver metastasis. Cancer Biol Ther 12: 69-79, 2011.

42. Georges R, Bergmann F, Hamdi H, et al: Sequential biphasic changes in claudin 1 and claudin 4 expression are correlated to colorectal cancer progression and liver metastasis. J Cell Mol Med 16: 260-272, 2012.

43. Wittmer A, Khazaie K and Berger MR: Quantitative detection of lac-Z-transfected CC531 colon carcinoma cells in an orthotopic rat liver metastasis model. Clin Exp Metastasis 17: 369-376, 1999.

44. Ewing J: The Problems of melanoma. Br Med J 2: 852-856, 1930.

45. Ewing J: Neoplastic diseases. Saunders-Verlag, Philadelphia, pp125-129, 1928

46. Paget S: The distribution of secondary growths in cancer of the breast. 1889. Cancer Metastasis Rev 8: 98-101, 1989.

47. Chishima T, Miyagi Y, Wang X, Yamaoka H, Shimada H, Moossa AR and Hoffman RM: Cancer invasion and micrometastasis visualized in live tissue by green fluorescent protein expression. Cancer Res 57: 2042-2047, 1997. 